\title{
Analysis of a Regulated Cascode Cross Couple Power Amplifier
}

\author{
Kittipong Tripetch \\ Division of Electronic and \\ Telecommunication engineering \\ Faculty of Engineering and \\ Architecture \\ Rajamangala University of \\ Technology Suvarnabhumi \\ Kittipong.tripetch.mr@ieee.org
}

\begin{abstract}
The input impedance of a regulated cascode cross couple amplifier is derived. The frequency response of the input impedance polynomial form can be plotted with MATLAB. From the polynomial form of the input impedance of the proposed circuit, it can be transformed by substitute complex frequency $\mathbf{s}$ with $j \omega$ into the polynomial form equation. After that, this function can be grouped into a symbolic real and a symbolic imaginary form. The next step in derivation is to multiply this function with a complex conjugate function of the symbolic complex form of the input impedance. The last step is to plot a real and an imaginary part as a function of the input frequency so that the power amplifier can be matching with the various matching circuit according to the condition of the maximum power transfer.
\end{abstract}

Keywords - a regulated cascode amplifier, input impedance, oscillator, power amplifier, a regulated cascode cross couple amplifier

\section{INTRODUCTION}

The Regulated Cascode amplifier was proposed by Sackinger since 1990 [1]. This topology of amplifier is famous because it has a higher output impedance compared with cascode amplifier counterpart. Thus, its output voltage is higher than other types of an amplifier such as a common source, a common gate and a cascode amplifier. The concept of the regulated transistor can be replaced by an amplifier block diagram which is used to regulated drain-source voltage drop of the first stage transistor but it used more transistor which was proposed since 1990 [2]. The advantage of this idea is a more voltage gain but the disadvantage is, it has more parasitic capacitances which degrade the bandwidth of the amplifier. The circuit technique called a regulated cascode also have many types of application like a switched current integrator which can be replaced in the signal flow graph of the high order switched current filter which was published since 1993 [3]. It can be used as the core circuit of current mirror. Its advantageous compared to other circuit technique is a fast settling time for a low value of current mirror between 10 microampere to 600 microampere. It is much slower than a simple current mirror if it is designed to operate for 530

\footnotetext{
Identify applicable funding agency here. If none, delete this text box.
}

microampere to 800 microampere. The Class $\mathrm{AB}$ regulated cascode current memory can be used as the core circuit of the switched current integrator. The Class $A B$ means it can be operated for half a cycle of a signal for each input transistor NMOS and PMOS. It is published since 1994 [4]. Other applications of the regulated cascode amplifier can be modified as a transconductance amplifier and transimpedance amplifier which are published during 1997-2015 [5]-[12].The tuning range of the regulated cascode cross couple oscillator can be approximated since 2013 [13]. Recent specifications which are related with a transimpedance amplifier based on RGC circuit diagram and its modification are published in [14]-[16]

In section II, a straightforward definition of input impedance and circuit diagram definition is described. A high frequency equivalent circuit is shown and described in Section III. In section IV, a lucent and rigorous detail procedure for circuit analysis of the regulated cascode cross coupled amplifier is proposed for the first time. In section $\mathrm{V}$, the graphs of input impedance as a function of input frequency and current consumption are plotted by programing with level1 transistor model. In section VI, the graphs of real part and imaginary part as a function of an input frequency and current consumption are plotted by programing with level1 transistor model.

\section{INPUT IMPEDANCE OF THE REGULATED CASCODE CROSS COUPLED AMPLIFIER DESCRIPTION}

The input impedance is a ratio of input voltage and input current. It can be derived by using Kirchhoff's current law and Ohm's law. The Circuit diagram of the regulated cascode cross couple amplifier is drawn in figure1. The high frequency equivalent circuit of MOSFET is used to substitute for circuit analysis purpose. It is shown in figure 2. The regulated cascode amplifier can be described as a cascade of a first stage common-source amplifier which used transistor $M_{1}$ and a common gate amplifier. A regulated transistor can be seen as a cascade of a second stage common source amplifier which used transistor $\mathrm{M}_{3}$ with a first stage common source amplifier. The output of the second stage of a common source amplifier can be cascaded with the cascode transistor $\mathbf{M}_{2}$. 


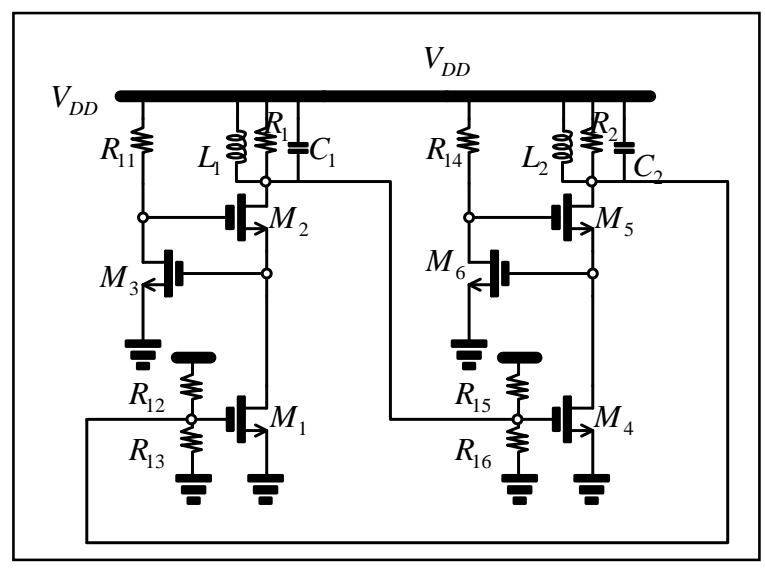

The regulated cascode cross coupled amplifier is shown in figure1. It composed of 6 NMOS transistors. The circuit has 4 bias resistors which are used to controlled drain current of the MOSFET. There are two resonance circuits which used two parallel inductor, capacitor and resistors. The output of the first regulated cascode amplifier is connected with the input of the second regulated cascode amplifier. The output of the second regulated cascode amplifier is feedback to the input of the first regulated cascode amplifier. That's why it is called the regulated cascode cross coupled amplifier. The high frequency equivalent circuit of the proposed circuit is shown in figure2. The two bulk source voltage controlled current source are included for the cascode transistors.

Fig. 1. The regulated cascode cross couple amplifier

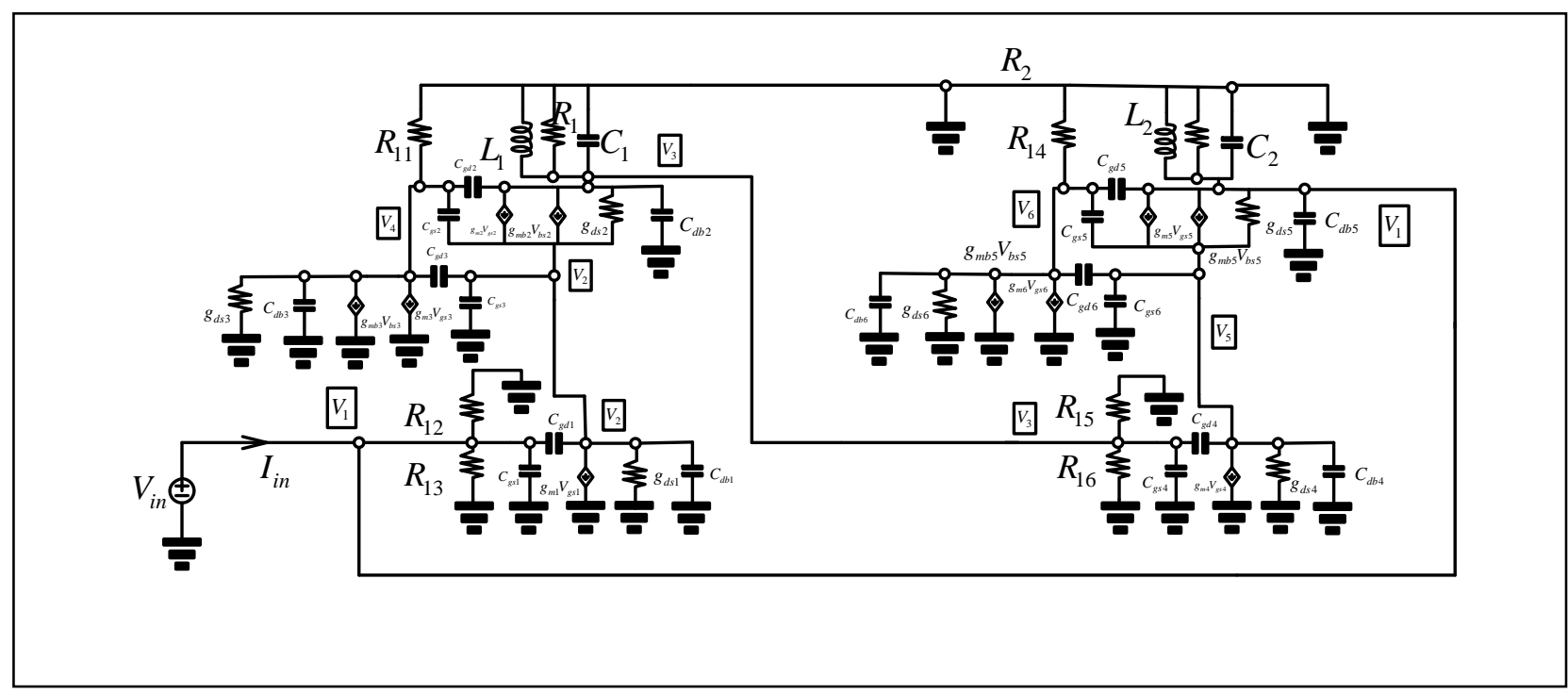

Fig. 2. The regulated cascode cross couple amplifier topology after substituted with a high frequency MOSFET equivalent circuit

\section{A HIGH FREQUENCY EQUIVALENT CIRCUIT OF A REGULATED CASCODE CROSS COUPLE AMPLIFIER DESCRIPTION}

The high frequency equivalent circuit of a regulated cascade cross couple amplifier is a crucial building block for circuit analysis and design purpose. It can be substituted with the circuit diagram in figure1. Some transistors used equivalent circuit elements only 5 elements. Some transistors used equivalent circuit elements for 6 elements. It is difference as a result of a fabrication process which has a bulk source voltage effect for a drain current can be modeled with voltage controlled current sourced which is labeled with $\mathrm{g}_{\mathrm{mb}}$ which can be pronounced as a transconductance of the bulk source voltage for a cascode transistor. There are six bias resistors in the circuit which can be labeled as $R_{11}$, $R_{12}, R_{13}, R_{14}, R_{15}$ and $R_{16}$. The total elements in the circuit can be counted to have 46 elements.

\section{A MATRIX OF KIRCHOFF'S CURRENT LAW (KCL) AND ITS COEFFICIENT DESCRIPTION}

There are six nodes in this circuit. It could be labeled as $\mathrm{V}_{1}, \mathrm{~V}_{2}, \mathrm{~V}_{3}, \mathrm{~V}_{4}, \mathrm{~V}_{5}$ and $\mathrm{V}_{6}$. After using Kirchhoff's current law and grouping the small signal parameters and its passive elements. It can be written as following.

$$
\left[\begin{array}{cccccc}
-b_{2} & 0 & 0 & 0 & b_{3} & b_{4} \\
b_{5} & -b_{6} & 0 & b_{7} & 0 & 0 \\
0 & a_{14} & -b_{8} & b_{9} & b_{10} & 0 \\
0 & b_{11} & b_{12} & -b_{13} & 0 & 0 \\
a_{25} & 0 & b_{14} & 0 & -b_{15} & b_{16} \\
b_{17} & 0 & 0 & 0 & b_{18} & b_{19}
\end{array}\right]\left[\begin{array}{c}
V_{1} \\
V_{2} \\
V_{3} \\
V_{4} \\
V_{5} \\
V_{6}
\end{array}\right]=\left[\begin{array}{c}
-I_{i n} b_{1} \\
0 \\
0 \\
0 \\
0 \\
0
\end{array}\right]
$$

$a_{i}$ and $b_{i}$ are novel coefficients which are a function of the small signal parameters and passive parameters in the regulated cascode cross couple amplifier. All novel coefficients could not be defined if it is duplicated the label of the coefficients. 


$$
\begin{aligned}
& a_{1}=L_{2} R_{12} R_{13} \\
& a_{2}=L_{2} R_{12} R_{13} C_{2}+L_{2} R_{12} R_{13} C_{g d 5}+L_{2} R_{12} R_{13} C_{d b 5} \\
& a_{3}=\frac{L_{2} R_{12} R_{13}}{R_{2}}+L_{2} R_{13}+L_{2} R_{12}+L_{2} R_{12} R_{13} g_{g d s} \\
& a_{4}=R_{12} R_{13} \\
& a_{5}=L_{2} R_{12} R_{13}\left(g_{m 5}+g_{m b 5}+g_{d s 5}\right) \\
& a_{6}=C_{g d 5} L_{2} R_{12} R_{13} \\
& a_{7}=g_{m 5} L_{2} R_{12} R_{13}
\end{aligned}
$$

$$
\begin{aligned}
& b_{1}=s a_{1} \\
& b_{2}=s^{2}\left(a_{2}\right)+s\left(a_{3}\right)+\left(a_{4}\right) \\
& b_{3}=s a_{5} \\
& b_{4}=s^{2}\left(a_{6}\right)-s\left(a_{7}\right)
\end{aligned}
$$

$$
\begin{aligned}
& a_{8}=C_{g d 1} \\
& a_{9}=g_{m 1} \\
& a_{10}=C_{g d 3}+C_{g s 2}+C_{g s 3}+C_{g d 1}+C_{d b 1} \\
& a_{11}=+g_{m 2}+g_{m b 2}+g_{d s 2}+g_{d s 1} \\
& a_{12}=s C_{g d 3}+s C_{g s 2}+g_{m 2} \\
& a_{13}=g_{m 2}
\end{aligned}
$$

$$
\begin{aligned}
& b_{5}=s a_{8}-a_{9} \\
& b_{6}=s a_{10}+a_{11} \\
& b_{7}=s a_{12}+a_{13}
\end{aligned}
$$

$$
\begin{aligned}
& a_{14}=s L_{1}\left(g_{m 2}+g_{m b 2}+g_{d s 2}\right) \\
& a_{15}=L_{1}\left(\frac{1}{R_{1}}+\frac{1}{R_{15}}+g_{d s 2}+\frac{1}{R_{16}}\right) \\
& a_{16}=L_{1}\left(C_{1}+C_{g d 4}+C_{g d 2}+C_{d b 2}+C_{g s 4}\right) \\
& a_{17}=\left(L_{1} C_{g d 2}\right), a_{18}=\left(L_{1} g_{m 2}\right), a_{19}=\left(L_{1} C_{g d 4}\right)
\end{aligned}
$$

$$
\begin{aligned}
& b_{8}=1+s a_{15}+s^{2}\left(a_{16}\right) \\
& b_{9}=s^{2} a_{17}-s a_{18} \\
& b_{10}=s^{2} a_{19}
\end{aligned}
$$

)

$$
\begin{aligned}
& a_{20}=\left(C_{g d 3}+C_{g s 2}\right) \\
& a_{21}=g_{m 3} \\
& a_{22}=C_{g d 2} \\
& a_{23}=\left[C_{g d 2}+C_{d b 3}+\left(C_{g d 3}+C_{g s 2}\right)\right] \\
& a_{24}=\left[\frac{1}{R_{11}}+g_{d s 3}\right]
\end{aligned}
$$

$$
\begin{aligned}
& b_{11}=\left[s a_{20}-a_{21}\right] \\
& b_{12}=\left(s a_{22}\right) \\
& b_{13}=\left(s a_{23}+a_{24}\right)
\end{aligned}
$$

$$
\begin{aligned}
& a_{25}=g_{d s 5}, a_{26}=C_{g d 4}, a_{27}=g_{m 4} \\
& a_{28}=\left(C_{g d 6}+C_{g s 5}+C_{g s 6}+C_{g d 4}+C_{d b 4}\right) \\
& a_{29}=\left(g_{m 5}+g_{m b 5}+g_{d s 5}+g_{d s 4}\right) \\
& a_{30}=\left(C_{g d 6}+C_{g s 5}\right), a_{31}=g_{m 5}
\end{aligned}
$$

$$
\begin{aligned}
& b_{14}=\left[s a_{26}-a_{27}\right] \\
& b_{15}=\left[s a_{28}+a_{29}\right] \\
& b_{16}=\left[s a_{30}+a_{31}\right]
\end{aligned}
$$

$$
\begin{aligned}
& a_{32}=\left(C_{g d 6}+C_{g s 5}\right) \\
& a_{33}=g_{m 6} \\
& a_{34}=C_{g d 5} \\
& a_{35}=\left[C_{g d 5}+C_{d b 6}+\left(C_{g d 6}+C_{g s 5}\right)\right] \\
& a_{36}=\left[\frac{1}{R_{14}}+g_{d s 6}\right]
\end{aligned}
$$




$$
\begin{aligned}
& b_{17}=s a_{32} \\
& b_{18}=s a_{33}-a_{34} \\
& b_{19}=s a_{35}+a_{36}
\end{aligned}
$$

All of the first stage intermediate coefficients which are written in the matrix of KCL are shown. From the matrix, it is a good time to describe how to eliminate column variables by writing $V_{1}=f\left(V_{5}, V_{6}, I_{i n}\right)$. There are four equations which are related with the first column. The starting equation is the first row. There are 3 equations which needs to eliminate the first variable which is called $V_{1}$. After substitute variable $V_{1}$ , the function can be grouped as following

$$
\begin{gathered}
d_{1}=\left(\frac{b_{3} b_{5}}{b_{2}}\right), d_{2}=\left(\frac{b_{4} b_{5}}{b_{2}}\right), d_{3}=\left(\frac{b_{1} b_{5}}{b_{2}}\right) \\
d_{4}=\left(\frac{b_{3} a_{25}}{b_{2}}-b_{15}\right), d_{5}=\left(\frac{b_{4} a_{25}}{b_{2}}+b_{16}\right), d_{6}=\left(\frac{b_{1} a_{25}}{b_{2}}\right) \\
d_{7}=\left(\frac{b_{3} b_{17}}{b_{2}}+b_{18}\right), d_{8}=\left(\frac{b_{4} b_{17}}{b_{2}}-b_{19}\right), d_{9}=\left(\frac{b_{1} b_{17}}{b_{2}}\right)
\end{gathered}
$$

The process of grouping after eliminate the first column variable is finished here. The second column is started as following. The function can be written as $V_{2}=f\left(V_{4}, V_{5}, V_{6}, I_{i n}\right)$ The system of equation can be update here so that the matrix in the single column can be seen as a single row left. It can be seen that the third and fourth row can be eliminate in the second column by substitute the function $V_{2}=f\left(V_{4}, V_{5}, V_{6}, I_{\text {in }}\right)$

$$
\left[\begin{array}{cccccc}
-b_{2} & 0 & 0 & 0 & b_{3} & b_{4} \\
0 & -b_{6} & 0 & b_{7} & d_{1} & d_{2} \\
0 & a_{14} & -b_{8} & b_{9} & b_{10} & 0 \\
0 & b_{11} & b_{12} & -b_{13} & 0 & 0 \\
0 & 0 & b_{14} & 0 & d_{4} & d_{5} \\
0 & 0 & 0 & 0 & d_{7} & d_{8}
\end{array}\right]\left[\begin{array}{c}
V_{1} \\
V_{2} \\
V_{3} \\
V_{4} \\
V_{5} \\
V_{6}
\end{array}\right]=\left[\begin{array}{c}
-I_{\text {in }} b_{1} \\
-I_{\text {in }} d_{3} \\
0 \\
0 \\
-I_{\text {in }} d_{6} \\
-I_{\text {in }} d_{9}
\end{array}\right]
$$

$$
\begin{gathered}
d_{10}=\left[b_{9}+\frac{b_{7} a_{14}}{b_{6}}\right], d_{11}=\left(b_{10}+\frac{d_{1} a_{14}}{b_{6}}\right) \\
d_{12}=\left(\frac{d_{2} a_{14}}{b_{6}}\right), d_{13}=\left(\frac{d_{3} a_{14}}{b_{6}}\right) \\
d_{14}=\left(\frac{b_{7} b_{11}}{b_{6}}-b_{13}\right), d_{15}=\left(\frac{d_{1} b_{11}}{b_{6}}\right) \\
d_{16}=\left(\frac{d_{2} b_{11}}{b_{6}}\right), d_{17}=\left(\frac{d_{3} b_{11}}{b_{6}}\right)
\end{gathered}
$$

The process of grouping after eliminate the second column variable is finished here. The third column variable can be eliminated by writing the function. It can be written as $V_{3}=f\left(V_{4}, V_{5}, V_{6}, I_{\text {in }}\right)$

$$
\begin{gathered}
{\left[\begin{array}{cccccc}
-b_{2} & 0 & 0 & 0 & b_{3} & b_{4} \\
0 & -b_{6} & 0 & b_{7} & d_{1} & d_{2} \\
0 & 0 & -b_{8} & d_{10} & d_{11} & d_{12} \\
0 & 0 & b_{12} & d_{14} & d_{15} & d_{16} \\
0 & 0 & b_{14} & 0 & d_{4} & d_{5} \\
0 & 0 & 0 & 0 & d_{7} & d_{8}
\end{array}\right]\left[\begin{array}{l}
V_{1} \\
V_{2} \\
V_{3} \\
V_{4} \\
V_{5} \\
V_{6}
\end{array}\right]=\left[\begin{array}{c}
-I_{i n} b_{1} \\
-I_{i n} d_{3} \\
-I_{\text {in }} d_{13} \\
-I_{\text {in }} d_{17} \\
-I_{\text {in }} d_{6} \\
-I_{\text {in }} d_{9}
\end{array}\right]} \\
d_{18}=\left[\frac{d_{10} b_{12}}{b_{8}+d_{14}}\right], d_{19}=\left[\frac{d_{11} b_{12}}{b_{8}+d_{15}}\right] \\
d_{20}=\left[\frac{d_{12} b_{12}}{b_{8}}+d_{16}\right], d_{21}=\left(\frac{d_{13} b_{12}}{b_{8}}+d_{17}\right) \\
d_{22}=\left[\frac{d_{10} b_{14}}{b_{8}}\right], d_{23}=\left[\frac{d_{11} b_{14}+d_{4}}{b_{8}}\right] \\
d_{24}=\left[\frac{d_{12} b_{14}}{b_{8}}+d_{5}\right], d_{25}=\left[\frac{d_{13} b_{14}}{b_{8}}+d_{6}\right]
\end{gathered}
$$

The process of grouping after eliminate the third column variable is finished here. The fourth column variable can be eliminated by writing the function. It can be written as $V_{4}=f\left(V_{5}, V_{6}, I_{\text {in }}\right)$ 


$$
\begin{aligned}
& {\left[\begin{array}{cccccc}
-b_{2} & 0 & 0 & 0 & b_{3} & b_{4} \\
0 & -b_{6} & 0 & b_{7} & d_{1} & d_{2} \\
0 & 0 & -b_{8} & d_{10} & d_{11} & d_{12} \\
0 & 0 & 0 & d_{18} & d_{19} & d_{20} \\
0 & 0 & 0 & d_{22} & d_{23} & d_{24} \\
0 & 0 & 0 & 0 & d_{7} & d_{8}
\end{array}\right]\left[\begin{array}{l}
V_{1} \\
V_{2} \\
V_{3} \\
V_{4} \\
V_{5} \\
V_{6}
\end{array}\right]=\left[\begin{array}{l}
-I_{\text {in }} b_{1} \\
-I_{\text {in }} d_{3} \\
-I_{\text {in }} d_{13} \\
-I_{\text {in }} d_{21} \\
-I_{\text {in }} d_{25} \\
-I_{\text {in }} d_{9}
\end{array}\right](23)} \\
& d_{26}=\left[d_{1}-\frac{d_{19} b_{7}}{d_{18}}\right], d_{27}=\left[d_{2}-\frac{d_{20} b_{7}}{d_{18}}\right], d_{28}=\left[\frac{d_{21} b_{7}}{d_{18}}-d_{3}\right]
\end{aligned}
$$

$$
\begin{aligned}
& d_{29}=\left[d_{11}-\frac{d_{19} d_{10}}{d_{18}}\right], d_{30}=\left[d_{12}-\frac{d_{20} d_{10}}{d_{18}}\right] \\
& d_{31}=\left[\frac{d_{21} d_{10}}{d_{18}}-d_{13}\right] \\
& d_{32}=\left[d_{23}-\frac{d_{19} d_{22}}{d_{18}}\right], d_{33}=\left[d_{24}-\frac{d_{20} d_{22}}{d_{18}}\right] \\
& d_{34}=\left[\frac{d_{21} d_{22}}{d_{18}}-d_{25}\right]
\end{aligned}
$$

The process of grouping after eliminate the fourth column variable is finished here. The fifth column variable can be eliminated by writing the function. It can be written as $V_{5}=f\left(V_{6}, I_{\text {in }}\right)$

$$
\begin{gathered}
{\left[\begin{array}{cccccc}
-b_{2} & 0 & 0 & 0 & b_{3} & b_{4} \\
0 & -b_{6} & 0 & 0 & d_{26} & d_{27} \\
0 & 0 & -b_{8} & 0 & d_{29} & d_{30} \\
0 & 0 & 0 & d_{18} & d_{19} & d_{20} \\
0 & 0 & 0 & 0 & d_{32} & d_{33} \\
0 & 0 & 0 & 0 & d_{7} & d_{8}
\end{array}\right]\left[\begin{array}{l}
V_{1} \\
V_{2} \\
V_{3} \\
V_{4} \\
V_{5} \\
V_{6}
\end{array}\right]=\left[\begin{array}{c}
-I_{i n} b_{1} \\
I_{i n} d_{28} \\
I_{\text {in }} d_{31} \\
-I_{\text {in }} d_{21} \\
I_{\text {in }} d_{34} \\
-I_{\text {in }} d_{9}
\end{array}\right]} \\
d_{35}=\left[\begin{array}{l}
\left.b_{4}-\frac{d_{33} b_{3}}{d_{32}}\right], d_{36}=\left[\frac{d_{34} b_{3}}{d_{32}}+b_{1}\right.
\end{array}\right] \\
d_{37}=\left[d_{8}-\frac{d_{33} d_{7}}{d_{32}}\right], d_{38}=\left[\frac{d_{34} d_{7}}{d_{32}}+d_{9}\right]
\end{gathered}
$$

The variable $\mathrm{V}_{5}$ of the first row and the sixth row can be eliminated from the fifth column. Thus, the last function to be written is $V_{6}=f\left(I_{i n}\right)=-I_{\text {in }}\left(\frac{d_{38}}{d_{37}}\right)$. This function can be used to eliminate the $\mathrm{V}_{6}$ variable so that the ratio of input impedance from the first row equation can be determined as

$$
\left[\begin{array}{cccccc}
-b_{2} & 0 & 0 & 0 & 0 & d_{35} \\
0 & -b_{6} & 0 & 0 & d_{26} & d_{27} \\
0 & 0 & -b_{8} & 0 & d_{29} & d_{30} \\
0 & 0 & 0 & d_{18} & d_{19} & d_{20} \\
0 & 0 & 0 & 0 & d_{32} & d_{33} \\
0 & 0 & 0 & 0 & 0 & d_{37}
\end{array}\right]\left[\begin{array}{c}
V_{1} \\
V_{2} \\
V_{3} \\
V_{4} \\
V_{5} \\
V_{6}
\end{array}\right]=\left[\begin{array}{c}
-I_{\text {in }} d_{36} \\
I_{\text {in }} d_{28} \\
I_{\text {in }} d_{31} \\
-I_{\text {in }} d_{21} \\
I_{\text {in }} d_{34} \\
-I_{\text {in }} d_{38}
\end{array}\right]
$$

The closed form equation of the input impedance can be derived as following

$$
Z_{\text {in }}=\frac{V_{1}}{I_{\text {in }}}=\left[d_{36}-\frac{d_{38} d_{35}}{d_{37}}\right]
$$

After closed form equation is finished. The polynomial form can be derived by considering the intermediate coefficients which have only 38 variables. It can be manipulate in the unpublished report but only the polynomial form results of each variable can be shown so that the space of the paper can be saved. All 38 variables of polynomial form which are done after substitute the function can be shown as following.

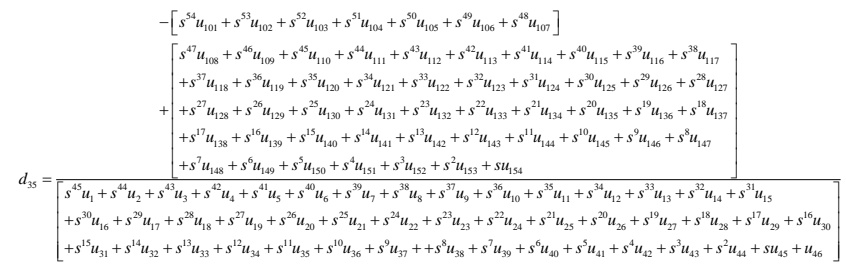

(32)

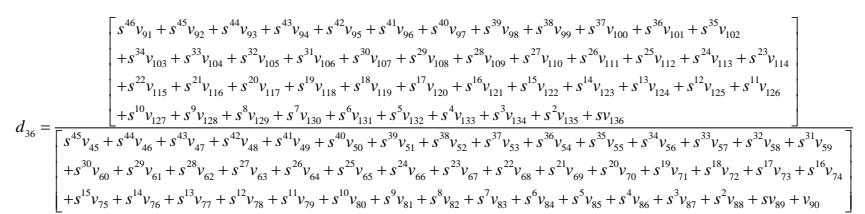

(33)

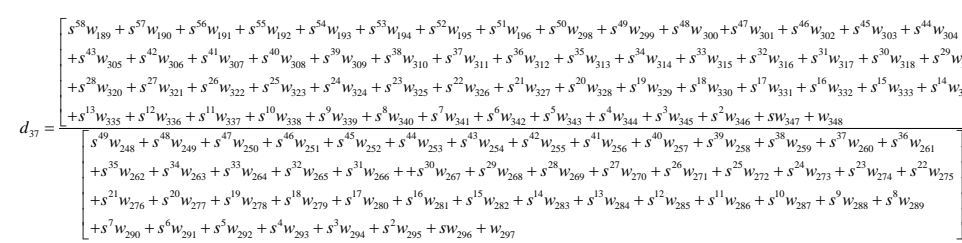

(34)

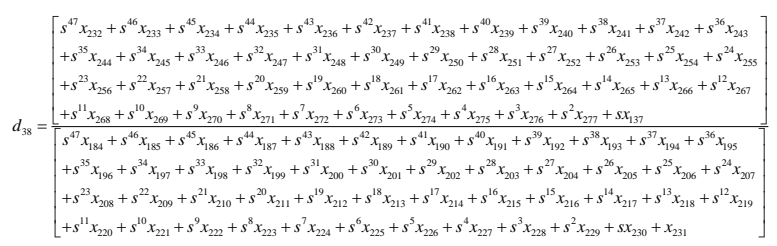


Table1 DC Operating Point Experimental Results Of the Regulated Cascode amplifier

\begin{tabular}{|c|c|c|c|c|c|}
\hline$R_{11}$ & $V_{R 11}$ & $V_{\text {OUT }}$ & $V_{G S, M_{2}}$ & $V_{D, M_{3}}$ & $V_{D S, M_{1}}$ \\
\hline & & & & & \\
\hline $46 \mathrm{k} \Omega$ & $308 \mathrm{mV}$ & $6.23 \mathrm{~V}$ & $93 \mathrm{mV}$ & $5.85 \mathrm{~V}$ & \\
\hline $100 \mathrm{k} \Omega$ & $1.89 \mathrm{~V}$ & $6.08 \mathrm{~V}$ & $84 \mathrm{mVac}$ & $2.23 \mathrm{~V}$ & $78 \mathrm{mVac}$ \\
\hline $220 \mathrm{k} \Omega$ & $1.00 \mathrm{~V}$ & $6.08 \mathrm{~V}$ & $0.20 \mathrm{Vac}$ & $4.87 \mathrm{~V}$ & $196 \mathrm{mVac}$ \\
\hline $430 \mathrm{k} \Omega$ & $1.36 \mathrm{~V}$ & $6.08 \mathrm{~V}$ & $0.15 \mathrm{Vac}$ & $4.58 \mathrm{~V}$ & $68 \mathrm{mVac}$ \\
\hline $510 \mathrm{k} \Omega$ & $1.67 \mathrm{~V}$ & $6.08 \mathrm{~V}$ & $0.16 \mathrm{Vac}$ & $4.48 \mathrm{~V}$ & $58 \mathrm{mVac}$ \\
\hline $681 \mathrm{k} \Omega$ & $2.05 \mathrm{~V}$ & $6.08 \mathrm{~V}$ & $87 \mathrm{mVac}$ & $4.14 \mathrm{~V}$ & $61 \mathrm{mVac}$ \\
\hline $910 \mathrm{k} \Omega$ & $1.961 \mathrm{~V}$ & $6.08 \mathrm{~V}$ & $0.18 \mathrm{Vac}$ & $3.89 \mathrm{~V}$ & $77 \mathrm{mVac}$ \\
\hline & & & & & \\
\hline
\end{tabular}

\section{A DESIGN OF A DC OPERATING POINT OF THE REGULATED CASCODE AMPLIFIER}

The DC operating point design is usually an iterative design process because there are more variables than the equations which is written from KCL and KVL of large signal drain current. From measurement the resonance circuit do not have voltage drop as a result of derivative of current of the supply voltage should be zero without ripple voltage. Thus, the input voltage bias of the second stage regulated cascode amplifier is not half of the supply voltage but it has approximately equal to the supply voltage of the battery.

\section{CONCLUSION}

The principles of eliminate column variable is clearly described. It is used to derive the ratio of input impedance of the regulated cascode cross couple power amplifier. The matrix coefficient update are used to show what is happened after the coefficients are grouped and defined many times so that it is easy to see which are the coefficient that can be eliminated in the next column. The column variable in the column matrix is left only one variable per column. The experimental result of a transistor array from ALD (ALD210802) is used to illustrate the difficulty to make a regulated transistor operating in the saturation region because of a drain to source voltage of the input transistor is too low. The input transistor is operating in the triode region.

MATLAB is used to execute the polynomial form of the input impedance after all of the coefficients are type into the text file with the level1 drain current model.

\section{REFERENCES}

[1] E. Sackinger, W. Guggenbuhl, “ A High-Swing, High-Impedance MOS Cascode Circuit”, IEEE Journal of Solid-State Circuits, Vol.25, No.1, February 1990, pp. 289-298

[2] K. Bult, G. J. G. M. Geelen, “ A Fast-settling CMOS Op Amp for SC Circuits with 90-dB DC Gain”, IEEE Journal of Solid-State Circuits, Nol.25, No.6, December 1990, pp. 1379-1384

[3] N. C Battersby and C. Toumazou, “ A $5^{\text {th }}$ order Bilinear Elliptic Switched-Current Filter", IEEE 1993 CICC, pp. 6.3.1- 6.3.4

[4] T. Serrano, B. Linares-Barranco, " The Active-Input RegulatedCascode Current Mirror", IEEE Transactinos on Circuits and System I: Fundamental Theory and Applications, Vol.41, No.6, June 1994, pp. 464-467

[5] A. H. Bratt, T. Olbrich and A.P. Dorey, "Class AB regulated cascode current memory cell", Electronics Letters, $27^{\text {th }}$ October 1994, Vol.30, No.22, pp. 1821-1822

[6] M. Goldenberg, R. Croman, T. S. Fiez, “Accurate SI Filters using RGC Integrators", IEEE Journal of Solid-State Circuits, Vol.11, November 1994, pp. 1388-1395

[7] D. Flandre, A. Viviani, J-P Eggermont, B. Gentinne, P. G. A. Jespers, "Improved Synthesis of Gain-Boosted Regulated-Cascode CMOS Stages using Symbolic Analysis and gm/ID Methodology, "IEEE Journal of Solid-State Circuits, Vol.32, No.7, July 1997, pp. 1006-1012

[8] M. Das, "Improved Design Criteria of Gain-Boosted CMOS OTA with High-Speed Optimizations", IEEE Transactinos on Circuits and Sytems II: Analog and Digital Signal Processing, Vol.49, No.3, March 2002, pp. 204-207

[9] S. M. Park, Hoi-Jun Yoo, "1.25 Gb/s Regulated Cascode CMOS Transimpedance Amplifier for Gigabit Ethernet Applications", IEEE Journal of Solid-State Circuits, Vol.39, No.1, January 2004, pp. 112121

[10] J. Nissinen, J. Kostamovaara, “ Fully Differential, Regulated Cascode Amplifier”, IEEE MELECON 2006, pp. 51-54

[11] Y. Zheng, C. E. Saavedra, " Feedforward-Regulated Cascode OTA for Gigahertz Applications", IEEE Transactions on Circuits and Systems I: Regular Papers, Vol.55, No.11, December 2008, pp. 3373-3382

[12] C. Li, S. Palermo, "A Low-Power $26 \mathrm{GHz}$ Transformer Based Regulated Cascode SiGe BiCMOS Transimpedance Amplifier", IEEE Journal of Solid-State Circuits, Vol. 48, No.5, May 2013, pp. 1264-1275

[13] K. Tripetch, "Comparative Analysis of Tuning Range of Regulated Cascode Cross Coupled CMOS Oscillator", Proceedings of the World Congress on Engineering 2013, Vol. II, WCE2013, July 3-5 2013, London, U. K.

[14] M H. Taghavi, L. Belostotski, James W. Haslett, P. Ahmadi, “ 10 $\mathrm{Gb} / \mathrm{s} \quad 0.13$ micron CMOS Inductorless Modified-RGC Transimpedance Amplifier", IEEE Transactinos on Circuits and Systems I: Regular Papers, Vol. 62, No.8, August 2015, pp. 19711980

[15] Oscal T.C. Chen, C-T Chan, Robin R-B Sheen, “ Transimpedance Limit Exploration and Inductor-Less Bandwidth Extension for Designing Wideband Amplifiers", IEEE Transactions on Very Large Integration (VLSI) Systems, Vol.24, No.1, January 2016, pp. 348352

[16] R. Costanzo, Steven M. Bowers, "A Current Resuse Regulated Cascode CMOS Transimpedance Amplifier with $11 \mathrm{GHz}$ Bandwidth", IEEE Microwave and Wireless Components Letters, Vol. 28, No.9, September 2018, pp. 816-818

\section{Creative Commons Attribution License 4.0 (Attribution 4.0 International, CC BY 4.0)}

This article is published under the terms of the Creative Commons Attribution License 4.0 https://creativecommons.org/licenses/by/4.0/deed.en_US 